\title{
Euler potentials for the MHD Kamchatnov-Hopf soliton solution
}

\author{
V. S. Semenov ${ }^{1}$, D. B. Korovinski ${ }^{1}$, and H. K. Biernat ${ }^{2}$ \\ ${ }^{1}$ Institute of Physics, State University, St. Petersburg, 198504 Russia \\ ${ }^{2}$ Space Research Institute, Austrian Academy of Sciences, Schmiedlstrasse 6, A-8042 Graz, Austria
}

Received: 28 August 2001 - Revised: 2 November 2001 - Accepted: 29 November 2001

\begin{abstract}
In the MHD description of plasma phenomena the concept of magnetic helicity turns out to be very useful. We present here an example of introducing Euler potentials into a topological MHD soliton which has non-trivial helicity. The MHD soliton solution (Kamchatnov, 1982) is based on the Hopf invariant of the mapping of a 3-D sphere into a 2-D sphere; it can have arbitrary helicity depending on control parameters. It is shown how to define Euler potentials globally. The singular curve of the Euler potential plays the key role in computing helicity. With the introduction of Euler potentials, the helicity can be calculated as an integral over the surface bounded by this singular curve. A special programme for visualization is worked out. Helicity coordinates are introduced which can be useful for numerical simulations where helicity control is needed.
\end{abstract}

\section{Introduction}

Magnetic helicity is a topological characteristic of magnetic field structures which includes the twisting and the kinking of a flux tube, as well as the linkage between different flux tubes (Moffatt, 1978; Biskamp, 1993). Among its numerous applications are dynamo theory (Moffatt, 1978), investigation of magnetic reconnection (Wiegelmann and Büchner, 2001), theory of relaxation (Taylor, 2000), and even the collimation mechanism of astronomical jets (Yoshizawa et al., 2000).

Magnetic helicity is defined as a volume integral

$K=\int_{\Omega}(\boldsymbol{A} \cdot \boldsymbol{B}) d^{3} x$,

where $\boldsymbol{B}$ is the magnetic field and $\boldsymbol{A}$ is the vector potential

$B=\nabla \times A$.

Correspondence to: $\mathrm{H}$. K. Biernat

(helfried.biernat@kfunigraz.ac.at)
Helicity (1) is gauge invariant, because under the transformation $\boldsymbol{A}^{\prime} \rightarrow \boldsymbol{A}+\nabla \phi$, it is then changed by

$\delta K=\int_{\Omega}(\nabla \phi \cdot \boldsymbol{B}) d^{3} x=\oint_{\partial \Omega} \phi(\boldsymbol{B} \cdot d \boldsymbol{S})=0$,

if $\left.B_{n}\right|_{\partial \Omega}=0$, where $\boldsymbol{n}$ is the vector normal to the boundary $\partial \Omega$. For $\left.B_{n}\right|_{\partial \Omega} \neq 0$, the surface integral does not vanish and the helicity becomes gauge dependent. Generally speaking, there is the possibility to define the helicity for the difference between the original field and the vacuum field (Schindler et al., 1988; Biskamp, 1993; Priest and Forbes, 2000) which helps to give the helicity a physical meaning for more realistic conditions. Nevertheless, we will restrict our consideration to the classical case $\left.B_{n}\right|_{\partial \Omega}=0$, leaving a more general definition of the magnetic helicity for future studies.

When the Euler potentials $\alpha, \beta$ are used,

$\boldsymbol{B}=\nabla \alpha \times \nabla \beta$,

there is the following problem related to helicity. It can be easily verified that

$\boldsymbol{A}=-\beta \nabla \alpha$

(or $\boldsymbol{A}=\alpha \nabla \beta$ ) is the vector potential (2) for the magnetic field (4). Then helicity vanishes at the level of the scalar product $(\boldsymbol{A} \cdot \boldsymbol{B})=0$. It is known (see, for example, Biskamp, 1993) that the vector potential can be presented in the following form (Clebsch representation)

$\boldsymbol{A}=-\beta \nabla \alpha+\nabla \psi$,

where the function $\psi$ (contrary to $\phi$ in Eq. 3) must be multivalued. This implies that the function $\psi$ has a surface $S_{j}$ inside the volume $\Omega$ where it has a jump, then the contribution from the jump surface $S_{j}$ is added to the integral over $\partial \Omega$ in Eq. (3) which results in the non-zero helicity.

The solution to the questions (a) how to introduce Euler potentials globally for the magnetic field with non-trivial helicity, (b) how to find the function $\psi$, and (c) why it has to be multi-valued, are unclear so far. For example, it is stated 
(Biskamp, 1993) that Euler potentials cannot be introduced globally for a magnetic field with non-zero helicity unless the system is multiply connected. In Sagdeev et al. (1986), it is pointed out that magnetic field lines determined by the Lagrangian invariants do not admit any linkage, i.e. the helicity has to vanish. The representation (5) is used sometimes (Priest and Forbes, 1999; Wong, 2000) quite generally, but it is not mentioned that helicity has to vanish in this case; hence, the structure of the magnetic field has to be relatively simple.

The aim of this paper is to show how one can introduce Euler potentials (4) in a practical way, as well as the Clebsch representation (6) for a magnetic field with non-zero helicity. There is a solution of the MHD equations (Kamchatnov, 1982; Sagdeev et al., 1986) based on the Hopf invariant of the mapping of a 3-D sphere $S^{3}$ into a 2-D sphere $S^{2}$ (see, for example, Dubrovin et al., 1979). In this solution, the magnetic flux tubes can be linked to each other as many times as one wants. The MHD soliton has a known helicity following from topology; hence, in each step of the calculation, there is an opportunity to control the situation. Besides, this solution is relatively simple, and all the results can be obtained analytically. We will not use topological methods, because all our results can be obtained straightforwardly if some topological information has been taken into account from the very beginning.

This paper is organized as follows. In Sects. 2 and 3, we recall the details of the MHD Kamchatnov-Hopf solution. Euler potentials are introduced in Sect. 4. A visualization of the magnetic field structure is presented in Sect. 5. Helicity coordinates are introduced in Sect. 6, and Sect. 7 is devoted to the summary and discussion.

\section{MHD Kamchatnov-Hopf soliton}

First of all, we will recall (Chandrasekhar, 1961; Kamchatnov, 1982) that any solinoidal vector field, $\operatorname{div} \boldsymbol{B}=0$, gives rise to a solution of the steady-state MHD equations

$\rho(\boldsymbol{v} \cdot \nabla) \boldsymbol{v}=-\nabla P+\frac{1}{4 \pi}(\boldsymbol{B} \cdot \nabla) \boldsymbol{B}$,

$(\boldsymbol{v} \cdot \nabla) \boldsymbol{B}=(\boldsymbol{B} \cdot \nabla) \boldsymbol{v}$,

$\operatorname{div} \boldsymbol{v}=0$,

$\operatorname{div} \boldsymbol{B}=0$,

in an incompressible plasma where the density $\rho=$ const. Here, $P$ is the total (gas + magnetic) pressure, and $v$ is the plasma velocity. If we choose $\boldsymbol{v}=\boldsymbol{B} / \sqrt{4 \pi \rho}$, and $P=$ const, then Eqs. (7-10) are satisfied automatically. In this solution, the magnetic tension is balanced by the centrifugal force.

The idea of the Kamchatnov-Hopf soliton solution is to obtain a solenoidal vector field with known linkage using topological methods.

A 3-D sphere $S^{3}$ is defined in $R^{4}$ as a set of points $\left(q_{1}, q_{2}, q_{3}, q_{4}\right)$, such that $q_{1}^{2}+q_{2}^{2}+q_{3}^{2}+q_{4}^{2}=1$. Let us introduce two complex numbers, $Z_{1}=q_{1}+i q_{2}, Z_{2}=q_{3}+i q_{4}$, then $S^{3}$ can also be described as $\left|Z_{1}\right|^{2}+\left|Z_{2}\right|^{2}=1$. A curve (a circle) on $S^{3}$ can be presented as

$\boldsymbol{l}(t)=\left(Z_{1} e^{i \omega_{1} t}, Z_{2} e^{i \omega_{2} t}\right)$,

where $t$ is a parameter along the curve. It can be shown (Dubrovin et al., 1979) that two curves corresponding to different initial points $Z_{1}, Z_{2}$ with integer numbers $\omega_{1}, \omega_{2}$ link each other $\omega_{1} \omega_{2}$ times.

A tangential field $\boldsymbol{Y}$ on $S^{3}$ generated by the curve (11) is

$\boldsymbol{Y}\left(\omega_{1}, \omega_{2}\right)=\frac{d \boldsymbol{l}(t)}{d t}=\left(-\omega_{1} q_{2}, \omega_{1} q_{1},-\omega_{2} q_{4}, \omega_{2} q_{3}\right)$,

which also has the linkage $\omega_{1} \omega_{2}$. Now we can map the curve (11) into $R^{3}$ using the stereographic projection

$x_{i}=\frac{q_{i}}{1+q_{4}}, \quad i=1,2,3$,

$q_{4}=\frac{1-x^{2}}{1+x^{2}}, \quad q_{i}=\frac{2 x_{i}}{1+x^{2}}, \quad i=1,2,3$.

To obtain the vector field (12) in $R^{3}$, we can just differentiate Eq. (13) with respect to parameter $t$

$$
\begin{aligned}
\boldsymbol{J}= & {\left[-\frac{4\left(\omega_{2} x_{1} x_{3}+\omega_{1} x_{2}\right)}{\left(x^{2}+1\right)^{2}}, \quad \frac{4\left(\omega_{1} x_{1}-\omega_{2} x_{2} x_{3}\right)}{\left(x^{2}+1\right)^{2}},\right.} \\
& \left.\frac{2 \omega_{2}\left(x_{1}^{2}+x_{2}^{2}-x_{3}^{2}-1\right)}{\left(x^{2}+1\right)^{2}}\right] .
\end{aligned}
$$

Stereographic projection conserves the topological invariant that is the linkage $\omega_{1} \omega_{2}$.

As a matter of fact, $\operatorname{div} \boldsymbol{J} \neq 0$, but it can be easily verified that the field $\boldsymbol{B}=\boldsymbol{J} /\left(1+x^{2}\right)$ is solenoidal, where $x^{2}=$ $x_{1}^{2}+x_{2}^{2}+x_{3}^{2}$. The factor $1 /\left(1+x^{2}\right) \neq 0$ exists everywhere in $R^{3}$, therefore, the field obtained,

$$
\begin{aligned}
\boldsymbol{B}= & \frac{2}{\left(1+x^{2}\right)^{3}}\left\{-2\left(\omega_{2} x_{1} x_{3}+\omega_{1} x_{2}\right),\right. \\
& \left.2\left(\omega_{1} x_{1}-\omega_{2} x_{2} x_{3}\right), \omega_{2}\left(x_{1}^{2}+x_{2}^{2}-x_{3}^{2}-1\right)\right\},
\end{aligned}
$$

has the same topological property as the field (12) on $S^{3}$.

The field (16) is the basis for the topological soliton. As was pointed out previously, if we introduce the plasma velocity $\boldsymbol{v}=\boldsymbol{B} / \sqrt{4 \pi \rho}$, and the pressure $P=$ const, then MHD Eqs. (7-10) are satisfied automatically. We will refer to this solution as the MHD Kamchatnov-Hopf soliton.

\section{Magnetic field lines}

Let us now derive the equation of the magnetic field lines in $R^{3}$. To this end, we can solve differential equations $d \boldsymbol{r} / d \lambda=\boldsymbol{B}$ using Eq. (16), but it is much easier just to map the known integral curves (11) from $S^{3}$ to $R^{3}$, with the help of stereographic projection (Kamchatnov, 1982; Sagdeev et al., 1986)

$$
x_{1}(t)=\frac{2\left(x_{10} \cos \left(\omega_{1} t\right)-x_{20} \sin \left(\omega_{1} t\right)\right)}{1+x_{0}^{2}+\left(1-x_{0}^{2}\right) \cos \left(\omega_{2} t\right)+2 x_{30} \sin \left(\omega_{2} t\right)},
$$




$$
\begin{aligned}
& x_{2}(t)=\frac{2\left(x_{20} \cos \left(\omega_{1} t\right)+x_{10} \sin \left(\omega_{1} t\right)\right)}{1+x_{0}^{2}+\left(1-x_{0}^{2}\right) \cos \left(\omega_{2} t\right)+2 x_{30} \sin \left(\omega_{2} t\right)}, \\
& x_{3}(t)=\frac{2 x_{30} \cos \left(\omega_{2} t\right)-\left(1-x_{0}^{2}\right) \sin \left(\omega_{2} t\right)}{1+x_{0}^{2}+\left(1-x_{0}^{2}\right) \cos \left(\omega_{2} t\right)+2 x_{30} \sin \left(\omega_{2} t\right)} .
\end{aligned}
$$

Using trigonometric identities, it is possible to reduce Eq. (17) to the following form

$$
\begin{aligned}
& x_{1}=\frac{\cos \Theta_{1}}{a+b \cos \Theta_{2}}, \\
& x_{2}=\frac{\sin \Theta_{1}}{a+b \cos \Theta_{2}}, \\
& x_{3}=\frac{b \sin \Theta_{2}}{a+b \cos \Theta_{2}},
\end{aligned}
$$

where

$$
\begin{aligned}
& \Theta_{1}=\omega_{1} t+\alpha_{1}, \quad \Theta_{2}=-\omega_{2} t+\alpha_{2}, \\
& a=\frac{1+x_{0}^{2}}{2 \sqrt{x_{10}^{2}+x_{20}^{2}}}, \quad b^{2}=a^{2}-1=\frac{4 x_{30}^{2}+\left(1-x_{0}^{2}\right)}{4\left(x_{10}^{2}+x_{20}^{2}\right)} \\
& \cos \alpha_{1}=\frac{x_{10}}{\sqrt{x_{10}^{2}+x_{20}^{2}}}, \quad \sin \alpha_{1}=\frac{x_{20}}{\sqrt{x_{10}^{2}+x_{20}^{2}}}, \\
& \cos \alpha_{2}=\frac{1-x_{0}^{2}}{\sqrt{4 x_{30}^{2}+\left(1-x_{0}^{2}\right)^{2}}}, \\
& \sin \alpha_{2}=\frac{2 x_{30}}{\sqrt{4 x_{30}^{2}+\left(1-x_{0}^{2}\right)^{2}}} .
\end{aligned}
$$

It turns out that the magnetic field lines lie on the surface of the torus

$$
\begin{aligned}
& x_{1}=\left(a+b \cos \Theta_{2}\right) \cos \Theta_{1}, \\
& x_{2}=\left(a+b \cos \Theta_{2}\right) \sin \Theta_{1}, \\
& x_{3}=b \sin \Theta_{2},
\end{aligned}
$$

which is produced by the rotation of the circle $x_{3}^{2}+\left(x_{1}-\right.$ $a)^{2}=a^{2}-1$ around the $x_{3}$ axis. The central torus degenerates into a circle (Sagdeev et al., 1986),

$x_{3}=0, \quad x_{1}^{2}+x_{2}^{2}=1$,

which will play an important role hereafter.

\section{Euler potentials}

It is convenient to choose as Euler potentials the following constants of integration (first integrals) of Eq. (19)

$$
\alpha=\alpha_{1} \omega_{2}+\alpha_{2} \omega_{1}, \quad \beta=\frac{1}{(2 a)^{2}},
$$

or, in Cartesian coordinates,

$$
\begin{gathered}
\alpha=\omega_{2} \arctan \frac{x_{2}}{x_{1}}+\omega_{1} \arctan \frac{2 x_{3}}{1-x^{2}}, \\
\beta=\frac{x_{1}^{2}+x_{2}^{2}}{\left(1+x^{2}\right)^{2}} .
\end{gathered}
$$

Then we can find the gradients of these functions

$$
\begin{aligned}
\nabla \alpha & =\left[\frac{4 \omega_{1} x_{1} x_{3}}{\left(x^{2}-1\right)^{2}+x_{3}^{2}}-\frac{\omega_{2} x_{2}}{x_{1}^{2}+x_{2}^{2}}, \frac{4 \omega_{1} x_{2} x_{3}}{\left(x^{2}-1\right)^{2}+x_{3}^{2}}\right. \\
& \left.+\frac{\omega_{2} x_{1}}{x_{1}^{2}+x_{2}^{2}},-\frac{2 \omega_{1}\left(x_{1}^{2}+x_{2}^{2}-x_{3}^{2}-1\right)}{\left(x^{2}-1\right)^{2}+x_{3}^{2}}\right], \\
\nabla \beta & =-\frac{2}{\left(1+x^{2}\right)^{3}}\left(x_{1}\left(x_{1}^{2}+x_{2}^{2}-x_{3}^{2}-1\right),\right. \\
& \left.x_{2}\left(x_{1}^{2}+x_{2}^{2}-x_{3}^{2}-1\right), 2 x_{3}\left(x_{1}^{2}+x_{2}^{2}\right)\right),
\end{aligned}
$$

and verify that Eq. (4) is satisfied, i.e. $\alpha, \beta$ are indeed, Euler potentials. The potential $\alpha$ is a naked angle (i.e. an angle that is not hidden under any trigonometric functions), which can have a non-zero contribution after integration of its gradient along a closed contour. Therefore, it is not surprising that first of all, $\alpha$ is a multi-valued function, and secondly, $\nabla \alpha$ has a singularity on the circle (21).

The next step is to obtain the vector potential $\boldsymbol{A}=-\beta \nabla \alpha$

$$
\begin{aligned}
\boldsymbol{A}= & \left\{\frac{-4 \omega_{1} x_{1} x_{3}\left(x_{1}^{2}+x_{2}^{2}\right)}{R}+\frac{\omega_{2} x_{2}}{\left(x^{2}+1\right)^{2}},\right. \\
& \frac{-4 \omega_{1} x_{2} x_{3}\left(x_{1}^{2}+x_{2}^{2}\right)}{R}-\frac{\omega_{2} x_{1}}{\left(x^{2}+1\right)^{2}}, \\
& \left.\frac{2 \omega_{1}\left(x_{1}^{2}+x_{2}^{2}\right)\left(x_{1}^{2}+x_{2}^{2}-x_{3}^{2}-1\right)}{R}\right\},
\end{aligned}
$$

where $R=\left(x^{2}+1\right)^{2}\left(\left(x^{2}-1\right)^{2}+4 x_{3}^{2}\right)$. Remember that the formal representation (5) leads to zero helicity, but we know that $K \neq 0$ by the topological construction, hence, the potential (26) should have some principal disadvantage. If vector potential $\boldsymbol{A}$ is defined by the differential Eq. (2), then we have to conclude that $\boldsymbol{A}$ is indeed the vector potential of the magnetic field $\boldsymbol{B}$, since Eq. (2) is satisfied. But besides the differential equation, there is also an integral equation

$\oint_{L}(\boldsymbol{A} \cdot d \boldsymbol{l})=\int_{S}(\boldsymbol{B} \cdot d \boldsymbol{S})=F_{B}$,

where $F_{B}$ is the magnetic flux through the surface $S$ bounded by the contour $L$. Differential and integral equations sometimes are not identical, and in our situation this is exactly the case. If we choose any contour $L$ which does not cross the disc bounded by the singular circle (21), then the circulation of $\boldsymbol{A}$ along $L$ gives exactly the magnetic flux $F_{B}$. However, if the contour encounters the disc bounded by singular circle (21), then the circulation obtains an additional contribution

$\oint_{L}(\boldsymbol{A} \cdot d \boldsymbol{l})=F_{B}+\frac{\pi \omega_{1}}{2}$,

therefore, the integral Eq. (27) is not satisfied. The formal reason for the multi-valued character of the circulation (28) lies in the singular behaviour of the latter at the circle (21), or due to the fact that the function $\alpha$ (22) is a naked angle. Hence, we have to proceed with the Clebsch representation (6), and to find a function $\psi$ to compensate the singularity in the potential (5). It is clear that the function $\psi$ also 


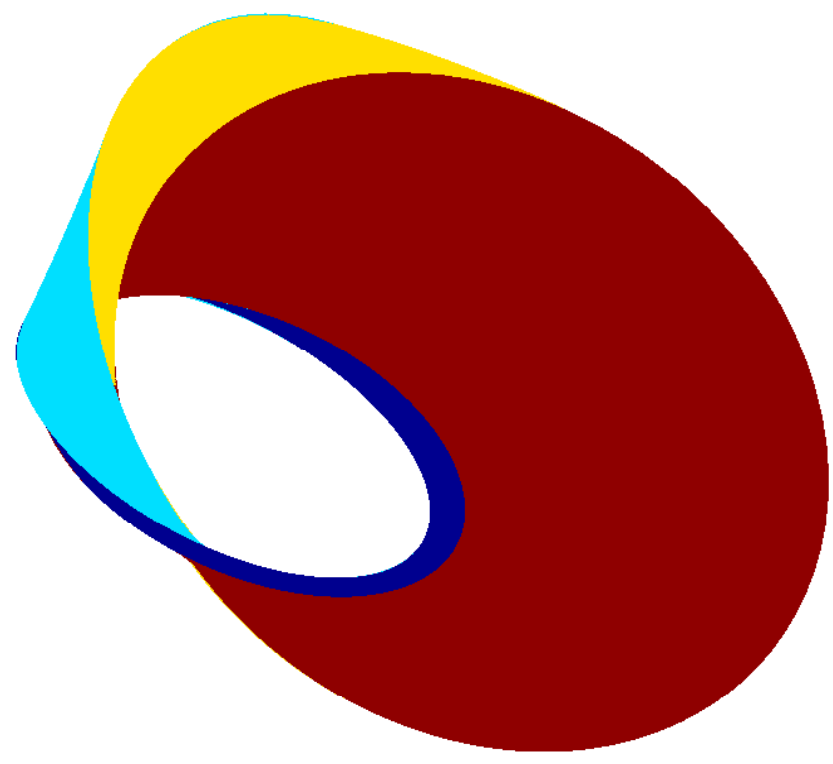

Fig. 1. Magnetic flux tube for the case $\omega_{1}=1, \omega_{2}=1$.

has to be a naked angle like the function $\alpha$, and its gradient should have a singularity at the circle (21)

$\psi=\frac{1}{4} \omega_{1} \arctan \left(\frac{x^{2}-1}{2 x_{3}}\right)=\frac{1}{4} \omega_{1}\left(-\omega_{2} t+\alpha_{2}+\frac{\pi}{2}\right)$.

Then, the Clebsch potential (6) turns out to be

$$
\begin{aligned}
\boldsymbol{A}= & \left\{\frac{\omega_{2} x_{2}+\omega_{1} x_{1} x_{3}}{\left(1+x^{2}\right)^{2}}, \quad \frac{\omega_{1} x_{2} x_{3}-\omega_{2} x_{1}}{\left(1+x^{2}\right)^{2}},\right. \\
& \left.\frac{\omega_{1}\left(1+x_{3}^{2}-x_{1}^{2}-x_{2}^{2}\right)}{2\left(1+x^{2}\right)^{2}}\right\} .
\end{aligned}
$$

It has no singularity in the whole space like the magnetic field (16), and both the differential (2) and the integral (27) equations are now satisfied.

It is interesting to note that the Clebsch representation (6) formally looks similar to the gauge condition $\boldsymbol{A}^{\prime} \rightarrow \boldsymbol{A}+\nabla \phi$. Nevertheless, there is an essential difference. The function $\phi$ has to be a single-valued one for the gauge transformation, at least for the simple connected region $\Omega$; hence, the integral of its gradient along any closed contour has to vanish. Contrary, the function $\psi$ in the Clebsch representation (6) has to be a multi-valued one, and the integral of its gradient along some closed contour can have non-zero contribution. Generally speaking, the question of whether the gauge function is a multi- or single-valued one is not really important for many applications in electrodynamics. But for such delicate characteristics of the field as the magnetic helicity, the solution of this question plays the key role. It is the multi-valued function $\psi$ that provides the non-zero helicity.

Using the vector potential (30) and the magnetic field (16), we can calculate the helicity as the volume integral (1)

$K=-\frac{\pi^{2} \omega_{1} \omega_{2}}{4}$.

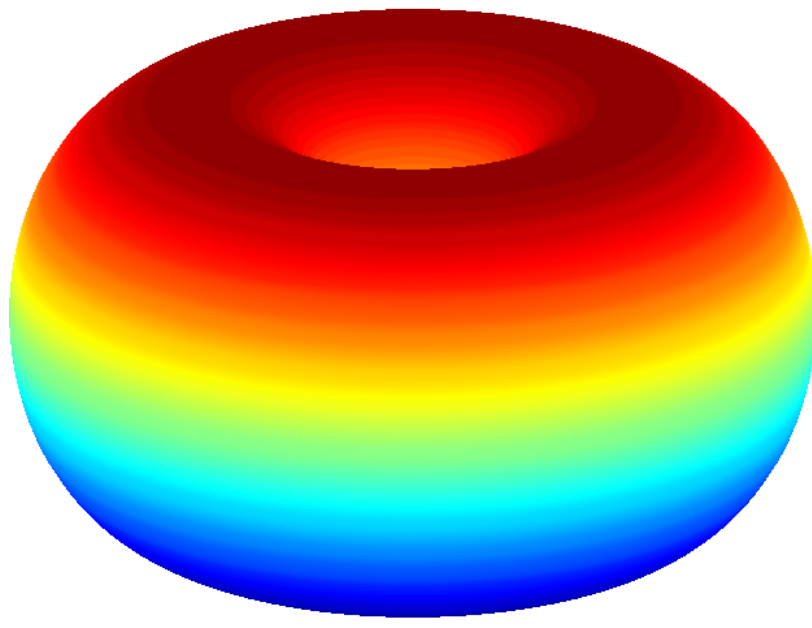

Fig. 2. Surface $\beta=$ const for the case $\omega_{1}=1, \omega_{2}=1$.

The negative sign in Eq. (31) is connected with the parameter $t$ in the initial curve (11) at $S^{3}$, so that $e^{i \omega t}$ gives a minus, whereas $e^{-i \omega t}$ gives a plus.

The Clebsch representation (6) leads to another way to compute the helicity

$K=\{\psi\} \int_{S}(\boldsymbol{B} \cdot d S)$,

which, of course, gives the same result (31). Here, $S$ is the singular circle (21), and $\{\psi\}$ is the jump of the function $\psi$ on the latter. As one can see, helicity can be calculated from the surface integral (32) rather than from the volume integral (1), which is simpler to do. It is also interesting that the helicity is equal to the magnetic flux through the singular circle times the jump of the $\psi$ function.

\section{Visualization}

It is worthwhile to present pictures of the magnetic field structure of the MHD Kamchatnov-Hopf soliton solution as mathematical examples for illustration. We start with the simplest case, $\omega_{1}=\omega_{2}=1$. The flux tube looks like a torus twisted by the angle $360^{\circ}$. To see this more clearly, the tube presented is chosen to have a rectangular cross section (Fig. 1), so that one can easily follow the screwed colour boundaries.

The surface Euler potential $\beta=$ const is just a usual torus (Fig. 2), since it stays more or less the same for all $\omega_{1}, \omega_{2}$. The magnetic field lines are swept around this torus.

The surface $\alpha=$ const is more complicated (Fig. 3). It is similar to a ribbon twisted by $360^{\circ}$. Such a surface cannot be continued to the closed one in $R^{3}$ without self crossing, because $\nabla \alpha$ has a singularity at the circle (21).

There is a simple way to imagine the magnetic field structure. Let us take a paper ribbon, twist it by the angle $360^{\circ}$ (note that twisting by $180^{\circ}$ gives a Moebius sheet), glue 


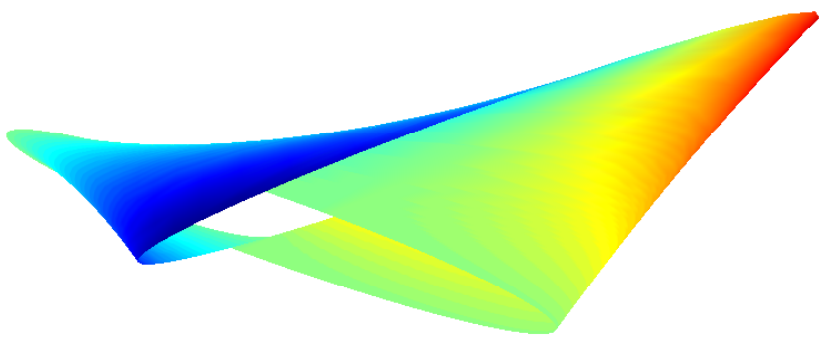

Fig. 3. Surface $\alpha=$ const for the case $\omega_{1}=1, \omega_{2}=1$.

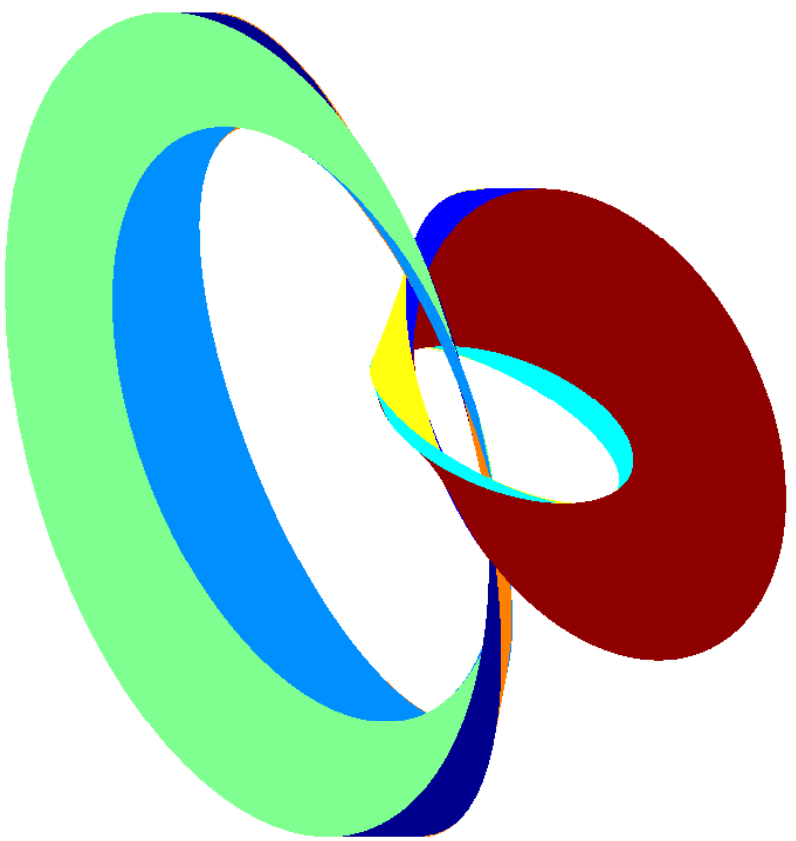

Fig. 4. Two linked flux tubes for the case $\omega_{1}=1, \omega_{2}=1$.

the edges of the ribbon together, and then cut it along the central line with scissors. As a result, we get two ribbons linked to each other. If we continue this procedure and cut the two ribbons obtained along their central axis, and so on, we can observe that each ribbon links any other one exactly one time (Fig. 4). This behaviour is reflected in the topological invariant helicity $K(31)$.

It is difficult to imagine that the intersection of two surfaces $\alpha=$ const and $\beta=$ const for different constants can give linked lines, nevertheless, it is so.

To complete the case $\omega_{1}=\omega_{2}=1$, we also present the surface $\psi=$ const (Fig. 5) which has a spiral structure converging to the singular circle (21).

After these relatively simple pictures we can proceed to the general case. First, we recall that two numbers are relatively prime if and only if the greatest common divisor of the numbers is one. For integers $\omega_{1}, \omega_{2}$ such that $\omega_{1}=n, \omega_{2}=m$ are relative prime, the magnetic field lines of the MHD Kamchatnov-Hopf soliton are linked into $(n, m)$ knots which are topologically nonequivalent for different $(n, m)$. They form the known family of toric nodes

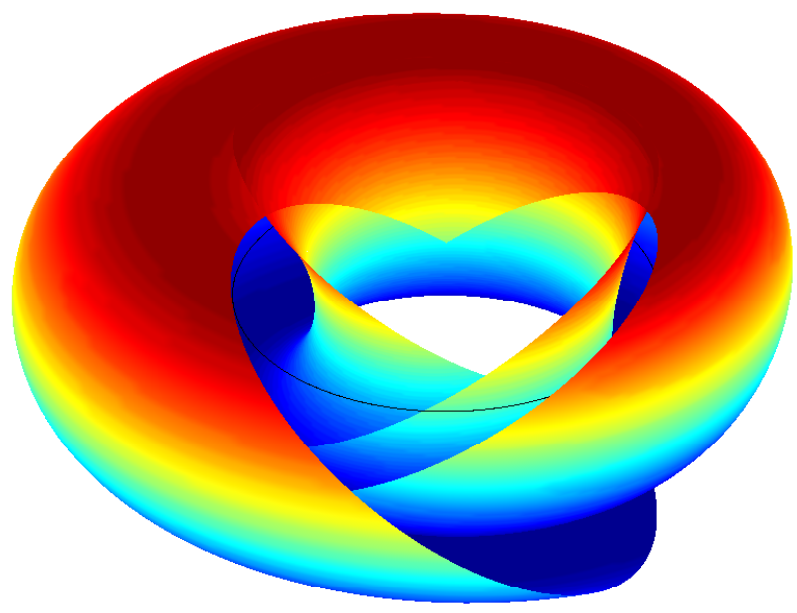

Fig. 5. Surface $\psi=$ const for the case $\omega_{1}=1, \omega_{2}=1$.

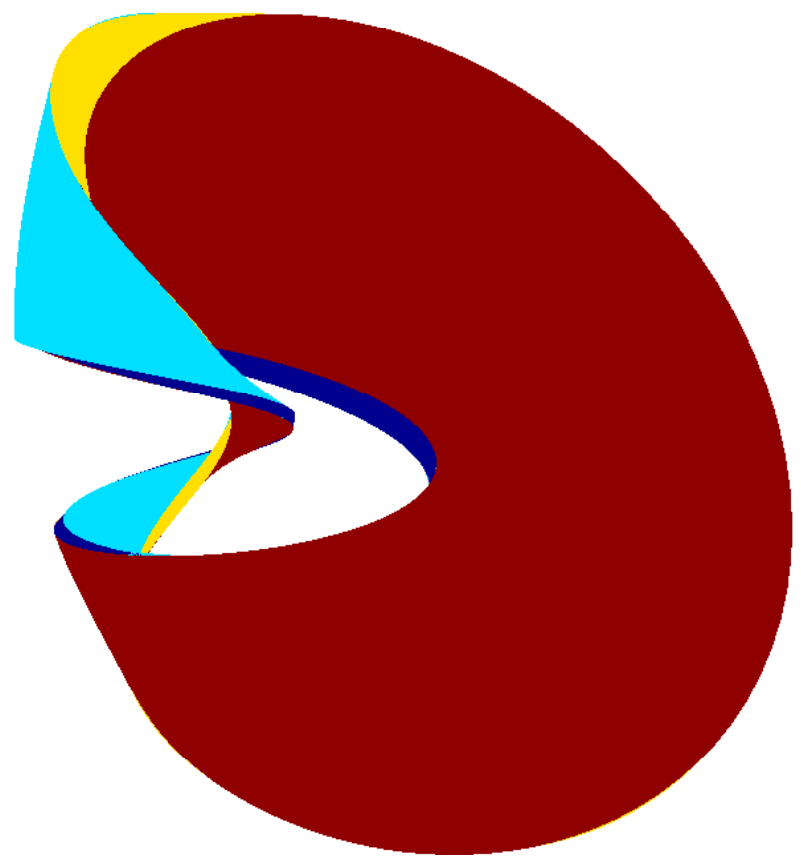

Fig. 6. Magnetic flux tube for the case $\omega_{1}=2, \omega_{2}=1$.

(Crowell and Fox, 1963).

The case $\omega_{1}=2, \omega_{2}=1$ is depicted in Fig. 6 (single flux tube), Fig. 7 (surface $\alpha=$ const), and Fig. 8 (knot 2,1).

The more complicated case $\omega_{1}=2, \omega_{2}=3$ is presented in Fig. 9 (single flux tube), Fig. 10 (surface $\alpha=$ const), Fig. 11 (central fragment of the surface $\alpha=$ const), and Fig. 12 (knot 2,3).

It is interesting that the surface of the Euler potential $\alpha=$ const for the latter case (Figs. 10, 11) is similar to a propeller, and this circumstance seems not to be a pure coincidence. The propeller has to create curls of air for producing a moving force, and at least some surfaces $\alpha=$ const (Figs. 3, 7, 10) might be used for this aim just from topological reasons. Of course, there is the question about the 


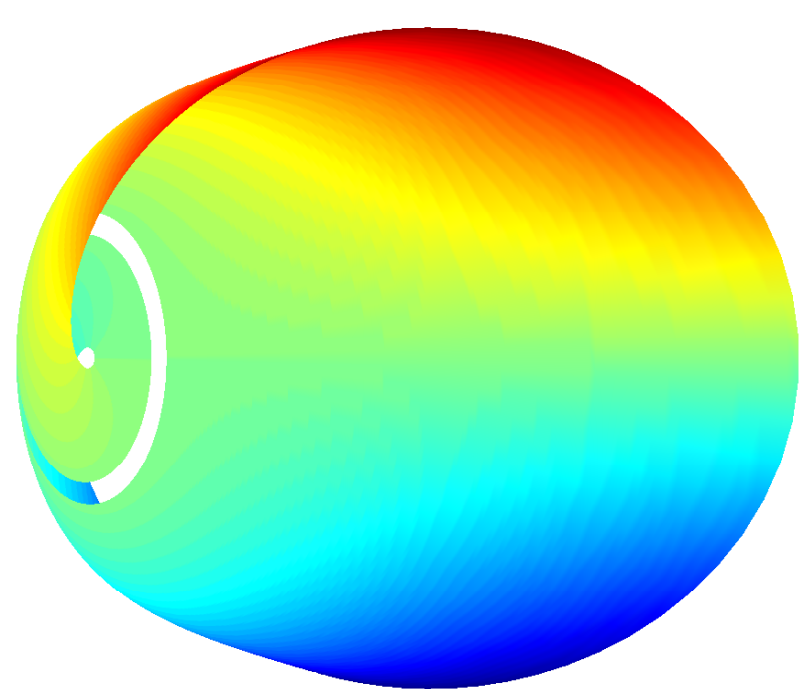

Fig. 7. Surface $\alpha=$ const for the case $\omega_{1}=2, \omega_{2}=1$.

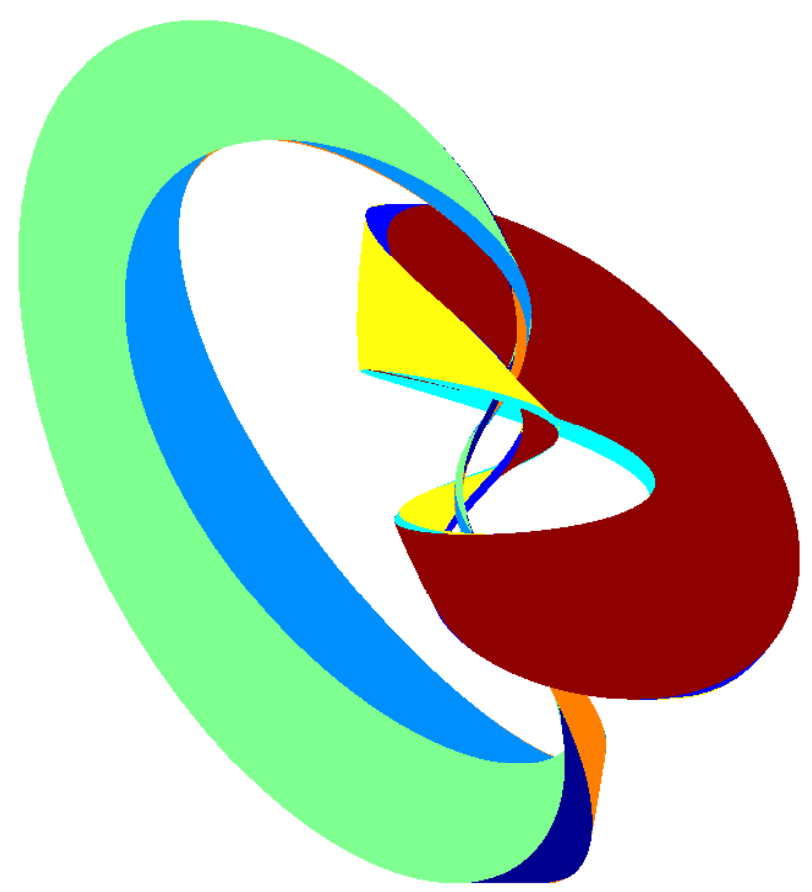

Fig. 8. Two linked flux tubes for the case $\omega_{1}=2, \omega_{2}=1$.

efficiency of such airscrews or waterscrews, but this is not the subject of this paper.

\section{Helicity coordinates}

A magnetic field line is defined by two Euler potentials $\alpha, \beta$, and a point on this line is controlled by the parameter $t$. We can use another parameter $\psi$ along the magnetic field line instead of $t$. Then $\alpha, \beta, \psi$, i.e. all functions taking part in the Clebsch representation of the vector potential (6), can be used as new curvilinear coordinates that have some useful property.

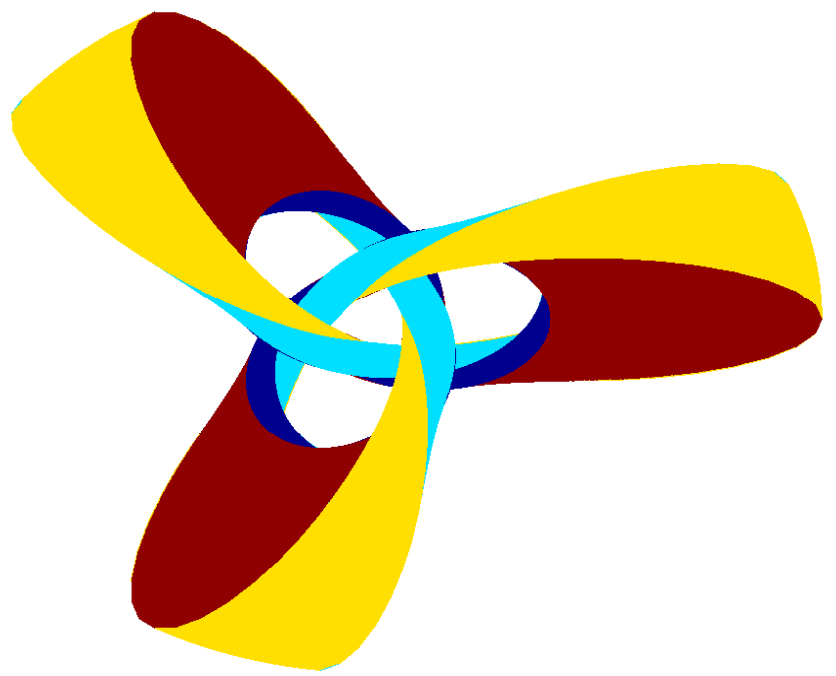

Fig. 9. Magnetic flux tube for the case $\omega_{1}=2, \omega_{2}=3$.

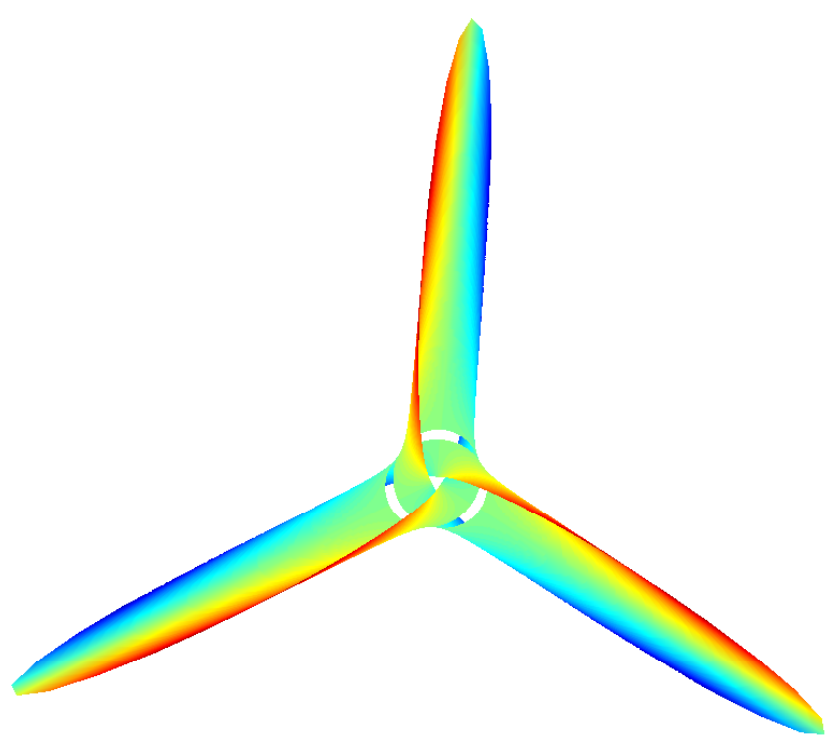

Fig. 10. Surface $\alpha=$ const for the case $\omega_{1}=2, \omega_{2}=3$.

We already have an expression for the Clebsch coordinates via Cartesian coordinates $(22,23,29)$. It is possible to simplify these equations, noting that without loss of generality, we can assume $\alpha_{1}=0$ in Eq. (22) and then obtain

$\alpha=\omega_{1} \arctan \frac{2 x_{3}}{1-x^{2}}$,

$\beta=\frac{x_{1}^{2}+x_{2}^{2}}{\left(1+x^{2}\right)^{2}}$,

$\psi=\frac{1}{4} \omega_{1} \arctan \left(\frac{x^{2}-1}{2 x_{3}}\right)$.

Now we can also find the mapping $\left(x_{1}, x_{2}, x_{3}\right) \rightarrow(\alpha, \beta, \psi)$

$x_{1}=\frac{2 \sqrt{\beta} \cos \left(\frac{\alpha}{\omega_{2}}+\frac{\pi \omega_{1}}{2 \omega_{2}}-\frac{4 \psi}{\omega_{2}}\right)}{\sqrt{1-4 \beta} \cos \left(\frac{4 \psi}{\omega_{1}}-\frac{\pi}{2}\right)+1}$, 


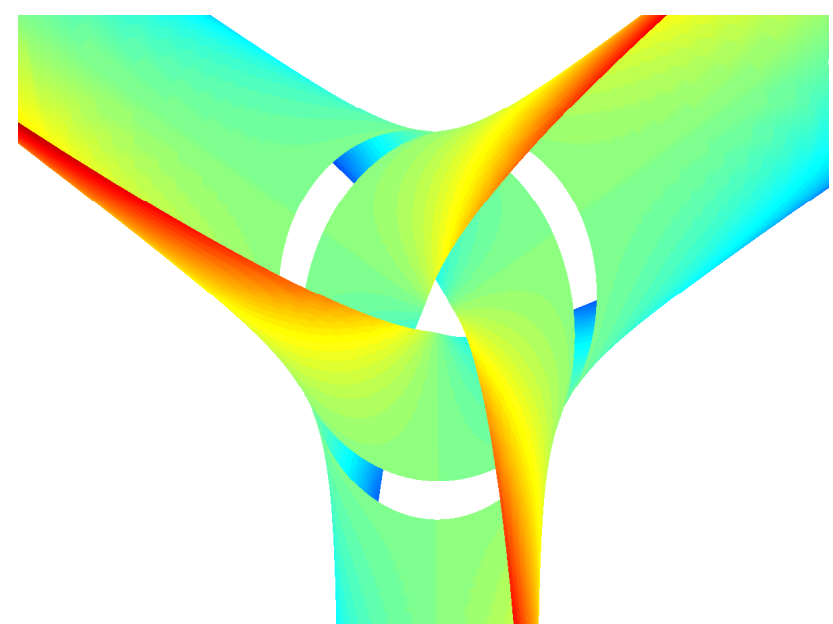

Fig. 11. Central fragment of the surface $\alpha=$ const for the case $\omega_{1}=2, \omega_{2}=3$.

$$
\begin{aligned}
& x_{2}=\frac{2 \sqrt{\beta} \sin \left(\frac{\alpha}{\omega_{2}}+\frac{\pi \omega_{1}}{2 \omega_{2}}-\frac{4 \psi}{\omega_{2}}\right)}{\sqrt{1-4 \beta} \cos \left(\frac{4 \psi}{\omega_{1}}-\frac{\pi}{2}\right)+1}, \\
& x_{3}=\frac{\sqrt{1-4 \beta} \sin \left(\frac{4 \psi}{\omega_{1}}-\frac{\pi}{2}\right)}{\sqrt{1-4 \beta} \cos \left(\frac{4 \psi}{\omega_{1}}-\frac{\pi}{2}\right)+1} .
\end{aligned}
$$

After some algebra one can find the Jacobian of this transformation

$$
J=\frac{D\left(x_{1}, x_{2}, x_{3}\right)}{D(\alpha, \beta, \psi)}=\frac{A}{B},
$$

where

$$
\begin{aligned}
A= & 8\left(\sqrt{(1-4 \beta)}+4 \beta \gamma^{2} \sqrt{(1-4 \beta)}+2(1-4 \beta)^{\frac{3}{2}} \gamma^{2}\right. \\
& -8 \beta \gamma+2 \gamma-\sqrt{\left.(1-4 \beta) \gamma^{2}\right)}, \\
B= & \omega_{2} \omega_{1}\left(-\gamma^{5}+12 \beta \gamma^{5}-48 \beta^{2} \gamma^{5}+64 \beta^{3} \gamma^{5}\right. \\
& -5(1-4 \beta)^{\frac{5}{2}} \gamma^{4}-10 \gamma^{3}+80 \beta \gamma^{3}-160 \beta^{2} \gamma^{3} \\
& \left.-10(1-4 \beta)^{\frac{3}{2}} \gamma^{2}-5 \gamma+20 \beta \gamma-\sqrt{(1-4 \beta)}\right), \\
\gamma= & \cos \left(-4 \frac{\psi}{\omega_{1}}+\frac{\pi}{2}\right) .
\end{aligned}
$$

This equation is a bit complicated, but nevertheless, it is possible to verify that $J \neq 0$ in the whole space, hence, the coordinates $(\alpha, \beta, \psi)$ can be introduced in $R^{3}$.

Let us compute the magnetic helicity using these new coordinates

$$
\begin{aligned}
K & =\int_{R^{3}}(\boldsymbol{A} \cdot \boldsymbol{B}) d x_{1} \wedge d x_{2} \wedge d x_{3} \\
& =\int_{R^{3}}(\nabla \psi \cdot[\nabla \alpha \times \nabla \beta]) d x_{1} \wedge d x_{2} \wedge d x_{3} \\
& =\int_{R^{3}} \frac{D(\alpha, \beta, \psi)}{D\left(x_{1}, x_{2}, x_{3}\right)} d x_{1} \wedge d x_{2} \wedge d x_{3}
\end{aligned}
$$

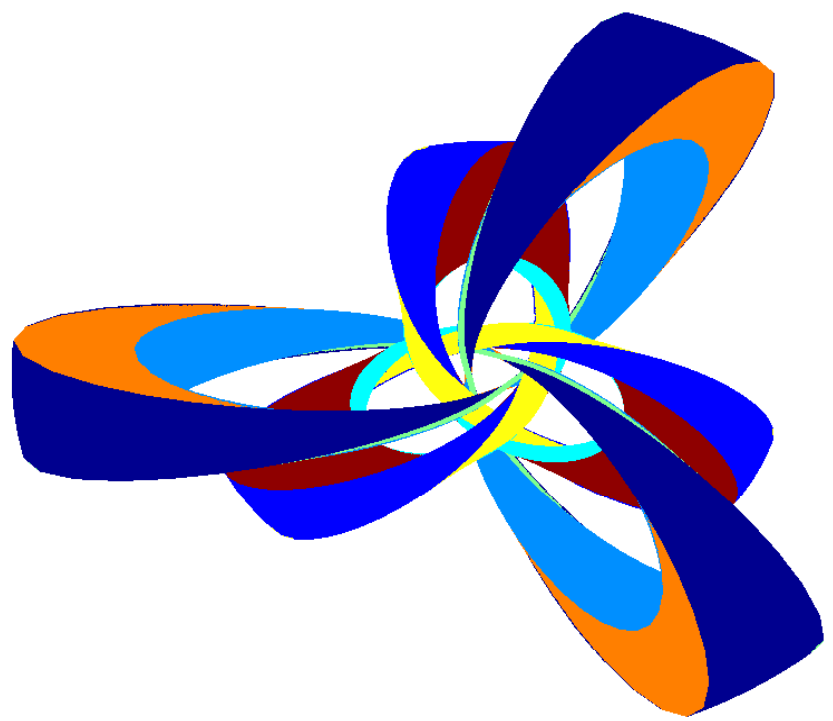

Fig. 12. Two linked flux tubes for the case $\omega_{1}=2, \omega_{2}=3$.

$$
=\int_{\Omega^{\prime}} d \alpha d \beta d \psi
$$

where it is assumed that the whole space is mapped into the region $\Omega^{\prime}, R^{3} \rightarrow \Omega^{\prime}$ under the transformation $\left(x_{1}, x_{2}, x_{3}\right) \rightarrow$ $(\alpha, \beta, \psi)$. Therefore, it turns out that in the new variables, magnetic helicity is equal to the volume of the configuration space $(\alpha, \beta, \psi)$. It is easy to verify that the new formula (37) gives the same result (31) for the helicity if we take into account that the coordinates $(\alpha, \beta, \psi)$ are varied within the following limits

$\alpha \in(-\pi, \pi] ; \quad \beta \in\left[0, \frac{1}{4}\right) ;$

$\psi \in\left(-\frac{1}{4} \omega_{1} \omega_{2} \pi, \frac{1}{4} \omega_{1} \omega_{2} \pi\right]$,

where brackets ( or [ are used to show that the element close to the bracket is excluded or included in the list of elements, respectively.

One can see that the space $R^{3}$ is mapped onto the parallelepiped (38), in which the straight lines ( $\alpha=$ const, $\beta=$ const) represent the magnetic field lines. It is surprising that the complicated magnetic structure is converted into a very simple geometrical object, that is the parallelepiped (38). In fact, the situation is not that simple. To make field lines which are closed, we have to glue the end points. The points on the left boundary $\alpha=-\pi$ have to be considered identical with those on the right boundary $\alpha=\pi$, and the points of the bottom boundary $\psi=-\frac{1}{4} \omega_{1} \omega_{2} \pi$ are identical with those on the upper boundary $\psi=\frac{1}{4} \omega_{1} \omega_{2} \pi$ after the rotation of the latter by the angle $2 \omega_{1} \omega_{2} \pi$.

We note that helicity coordinates can be particularly important for numerical simulations where helicity control is required. 


\section{Discussion and summary}

It was shown that Euler potentials can be introduced globally for a magnetic field with non-zero helicity even for the simply connected region (the space $R^{3}$ in our case), contrary to the remark of Biskamp (1993). Therefore, most of the coordinate systems (Pudovkin and Semenov, 1985; Pustovitov, 1999) based on Euler potentials (4), such as the helicity system (34), can also still be applied to magnetic structures with non-zero helicity $K \neq 0$. On the other hand, one has to be particularly careful with the vector potential. Remember that the simple representation (5) is not appropriate for the magnetic field with $K \neq 0$, instead the Clebsch representation (6) has to be used.

As we have seen, the function $\psi$ plays a key role in calculating the magnetic helicity. The multi-valued character of this function is connected with the singular behaviour of the gradient of at least one Euler potential ( $\alpha$ in our case). In its turn, the singularity of the Euler potential is the consequence of the fact that the $\alpha=$ const surface is highly twisted for the case $K \neq 0$ and cannot be continued to a closed surface in $R^{3}$.

The helicity turns out to be equal to the magnetic flux through the singular circle times the jump of the function $\psi$, hence, the calculation of $K$ can be reduced to a surface integral. It seems that the simple formula (32) can be extended to the general case as

$K=\sum_{j}\left\{\psi_{j}\right\} F_{B j}$,

where $F_{B j}$ is the magnetic flux through the surface bounded by the $j$-singular curve of the Euler potential and $\left\{\psi_{j}\right\}$ is the jump of the function $\psi$ at this surface.

If, by chance, it is known that all singular lines of the Euler potentials (separatrices) lie on a surface $S$ (the surface of the Sun, for example), then the magnetic helicity can be found using only data of the normal component of the magnetic field $B_{n}$ on $S$ from Eqs. (32) or (39), which is an important problem for solar physics. But if a singular line is inside the Sun, it is not possible to find the helicity using surface data. The maximum of what can be done is to estimate the helicity if one could somehow control the magnetic flux closed under the Sun's surface.

The Kamchatnov-Hopf solution seems to be the simplest one that can describe the magnetic field with such a nontrivial helicity. Therefore, it may play the same role for the investigation of different helicity problems as the Harris (1962) layer in plasma physics or the Petschek (1964) solution in reconnection theory.

Acknowledgement. We are grateful to I. V. Kubyshkin for helpful discussions. Part of this work was done while VSS and DBK were on a research visit to Graz. This work is partially supported by the Russian Foundation of Basic Research, grant No. 01-05-64954, by the INTAS-ESA grant No. 99-01277, and by the programme INTERGEOPHYSICS from the Russian Ministry of Higher Education. Part of this work is supported by the Austrian "Fonds zur Förderung der wissenschaftlichen Forschung", project P13804TPH. We acknowledge support by the Austrian Academy of Sciences, "Verwaltungsstelle für Auslandsbeziehungen".

\section{References}

Biskamp, D.: Nonlinear magnetohydrodynamics, Cambridge University Press, 1993.

Chandrasekhar, S.: Hydrodynamic and hydromagnetic stability, Oxford University Press, 1961.

Crowell, R. and Fox, R.: Introduction to knot theory, New York, 1963.

Dubrovin, B. A., Novikov, S. P., and Fomenko, A. T.: Modern geometry, Nauka, Moscow, 1979.

Harris, E. G.:, On a plasma sheath separating regions of oppositely directed magnetic fields, Nuovo Cimento, 23, 115-121, 1962.

Kamchatnov, A. M.: Topological soliton in magnetohydrodynamics, Sov. JETP, 82, 117-124, 1982.

Moffat, H. K.: Magnetic field generation in electrically conducting fluids, Cambridge University Press, 1978.

Petschek, H. E.: Magnetic field annihilation, NASA Spec. Publ., SP-50, 425-439, 1964.

Priest, E. and Forbes, T.: Magnetic reconnection, Cambridge University Press, 2000.

Pudovkin, M. I. and Semenov, V. S.: Magnetic field reconnection theory and the solar wind-magnetosphere interaction: A review, Space Sci. Revs., 41, 1-89, 1985.

Pustovitov, V. D.: Magnetic coordinates with double straightening, Plasma Phys. Rep., 25, 963-968, 1999.

Sagdeev, R. Z., Moiseev, S. S., Tur, A. V., and Yanovskii, V. V.: Problems of the theory of strong turbulence and topological soliton, in: Nonlinear Phenomena in Plasma Physics and Hydrodynamics, (Ed) Sagdeev, R. Z., MIR Publishes, Moscow, 135-158, 1986.

Schindler, K., Hesse, M., and Birn, J.: General magnetic reconnection, parallel electric fields and helicity, J. Geophys. Res., 93, 5547-5557, 1988.

Taylor, J. B.: Relaxation revisited, Phys. Plasmas, 7, 1623-1629, 2000.

Wiegelmann, T. and Büchner, J.: Evolution of magnetic helicity in the course of kinetic magnetic reconnection, Nonlin. Proc. Geophys., 8, 127-140, 2001.

Wong, H. V.: Particle canonical variables and guiding center Hamiltonian up to second order in the Larmor radius, Phys. Plasmas, 7, 73-83, 2000.

Yoshizawa, A., Yokoi, N., and Kato, H.: Collimation mechanism of magnetohydrodynamic jets based on helicity and cross-helicity dynamos, with reference to astronomical jets, Phys. Plasmas, 7, 2646-2653, 2000. 